Mon. Not. R. Astron. Soc. 000,11-?? (2005) ～Printed 11 November $2018 \quad$ (MN LTEX style file v2.2)

\title{
3C 40 in Abell 194 : \\ Can tail radio galaxies exist in a quiescent cluster?
}

\author{
Irini Sakelliou $^{1}$, M.J. Hardcastle ${ }^{2}$, and N.N. Jetha ${ }^{3,4}$ \\ ${ }^{1}$ Max-Planck-Institute für Astronomie, Königstuhl 17, D-69117, Heidelberg, Germany \\ ${ }^{2}$ School of Physics, Astronomy and Mathematics, University of Hertfordshire, College Lane, Hatfield AL10 9AB, UK \\ ${ }^{3}$ School of Physics and Astronomy, University of Birmingham, Edgbaston, Birmingham B15 2TT \\ ${ }^{4}$ CEA/Saclay, Service d'Astrophysique, L'Orme des Merisiers, Bât. 709, 91191 Gif-sur-Yvette Cedex, France
}

11 November 2018

\begin{abstract}
The nearby cluster Abell 194 hosts two luminous, distorted radio galaxies. Both reside within the cluster's core region, being separated in projection by only $100 \mathrm{kpc}$. It is often suggested that tailed radio galaxies such as these reside in clusters that are under formation and are accreting new material from their outskirts. In this paper we study the intriguing appearance of Abell 194, and test whether the cluster and radio source dynamics are consistent with the cluster formation/merger model. We analyse data from the XMM-Newton satellite and previously unpublished observations with the Very Large Array (VLA), as well as presenting new data from the Giant Metre-Wave Radio Telescope (GMRT).

The shape of the jets, and the lack of significant stripping of the galaxies' interstellar media, indicate that the radio galaxies are not moving at the large velocities they would have had if they were falling into the cluster from its outskirts; galaxy velocities of $\leqslant 300 \mathrm{~km} \mathrm{~s}^{-1}$ are adequate instead. A plausible scenario that could explain the observations is that the dynamics of the cluster centre are relatively quiescent, with the dominant system of massive galaxies being bound and orbiting the cluster centre of mass. For plausible jet/plume speeds and densities and the galaxy dynamics implied by this picture of the cluster, we show that the observed jet structures can be explained without invoking a major cluster merger event.
\end{abstract}

Key words: X-rays : galaxies : clusters - intergalactic medium - galaxies : clusters : individual (Abell 194)

\section{INTRODUCTION}

Radio galaxies in clusters normally have distorted morphologies, due to the interactions of the jets with the intracluster medium (ICM). In particular, the jets or plumes of two classes of radio galaxies that are found in groups or clusters of galaxies [the wideangle tail (WAT), and the Narrow-angle tail (NAT) radio galaxies] are bent symmetrically into wide or narrow $\mathrm{C}$-shapes. It is now believed that this particular shape is a result of the ram pressure exerted onto the jets and plumes as the host galaxy moves relative to the ICM. In this case, the bending direction of the tails should indicate the direction in which the host galaxy is moving. This information has been used in the past to study the motion of galaxies in clusters. For example, O'Dea et al. (1987), by measuring the orientation of the tails in NATs, found that the radio galaxies were associated with cluster galaxies in isotropic orbits, while Sakelliou \& Merrifield (2000) found that the WATs in their sample were mainly on radial orbits heading towards or away from the cluster centre.

Additionally, it has been suggested that both types of tailed sources are hosted by merging clusters (e.g., Pinkney, Burns \& Hill 1994). This was believed to be required in order that the galaxy velocities relative to the ICM should be large enough to produce the observed bending of the radio tails. However, this argument depends on the composition of the jet or plume. If the material in the plumes of WATs is light with respect to the surrounding medium (e.g, Hardcastle, Sakelliou \& Worrall 2005), the bending of the tails can be produced even with low galaxy velocities.

One of the rare clusters hosting more than one radio galaxy close to the cluster centre is Abell 1941. In this paper we use X-ray and radio observations of its core region to explore the dynamics of the cluster and its radio galaxies. In Sect. 2 we discuss the properties of the cluster, based mainly on X-ray data obtained with the XMM-Newton satellite. We present the radio data and discuss the characteristics of the radio galaxies in Sect. 3. Finally, in Sect. 4, we discuss a possible dynamical scenario that can give a good description of the data.

\footnotetext{
1 Abell 194 is at $\mathrm{z}=0.018$ (NED). Throughout this paper we use $H_{0}=$ $71 \mathrm{~km} \mathrm{~s}^{-1} \mathrm{Mpc}^{-1}, \Omega_{\mathrm{M}}=0.3$, and $\Omega_{\Lambda}=0.7$, giving a scale of $0.361 \mathrm{kpc}$ $\operatorname{arcsec}^{-1}$
} 


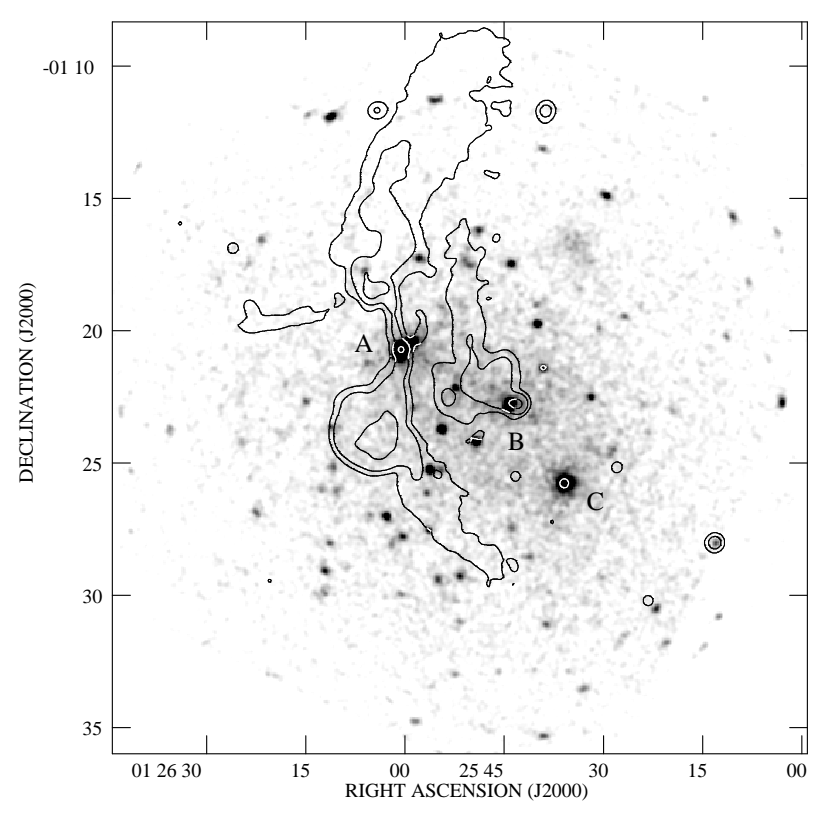

Figure 1. The XMM-Newton image of the cluster overlaid by the radio contours. The bright sources A, B, C are marked (see text).

\section{THE ABELL 194 CLUSTER}

Being at a low redshift, Abell 194 has been the subject of a number of investigations and the target of observations in wavelengths ranging from the optical to the X-rays. All past studies have found that it is a poor and cold cluster of richness $R=0$. One of its intriguing properties is that it appears as a 'linear cluster'. Nikogossyan et al. (1999) used the then available optical galaxy and ROSAT data to derive and compare the structural parameters of the galaxy and ICM distributions. They found that both distributions have similar ellipticities and position angles. Such a discovery could naturally be interpreted as showing that Abell 194 has been recently assembled by the accretion of smaller mass units along filaments, making it a recent merger remnant. However, searches for signatures of sub-structure related to a recent merging activity have proved unsuccessful. To our knowledge, both Lazzati et al. (1998) and Nikogossyan et al. (1999) applied a wavelet analysis to the ROSAT images, and detected X-ray emission from some cluster galaxies and some background groups or clusters of galaxies that appear at projected radii $>20$ arcmin from the core region of Abell 194 . But localized, enhanced X-ray emission, which would indicate the presence of dense clouds of gas within the cluster, was not found.

As mentioned above, the linear appearance of the cluster is also apparent in the X-ray images, and, as seen in the image of Fig. 1 this impression is given mainly by the presence of the X-ray sources $\mathrm{A}, \mathrm{B}, \& \mathrm{C}$ in a line. These sources were also present in past X-ray images (e.g., Nikogossyan et al. 1999).

Figure 1 comes from the XMM-Newton data, and it shows counts in the (0.5-5.0) keV energy range. Abell 194 was observed by the XMM-Newton satellite for a total of $\sim 22 \mathrm{ksec}$ on 2002 December 23-24 (revolution=557). During the observation the EPIC instruments were operating in the PrimeFullWindow (for MOS1 and MOS2), and PrimeFullWindowExtended (PN), and the thin filter was used for all imaging detectors. We obtained the raw XMMNewton data from the data archive, and processed them with SAS v6.5.0. EMCHAIN and EPCHAIN were used to obtain the calibrated event lists for the MOS and PN instruments respectively. The calibrated events were filtered for flags, using the XMM-Newton flags \#XMMEA_EM and \#XMMEA_EP for the two MOS and the PN detectors respectively. Restrictions on the pattern were also applied: we kept only events with pattern $<12$ for the MOS cameras, and $<4$ for the PN. We also cleaned the event lists for periods of high background, by applying a $3 \sigma$ clipping to the (10-15) keV lightcurves of each detector. This cleaning process reduced the exposure times by $\sim 2 \mathrm{ksec}$. For all the spectral analysis, we used the clean and filtered event lists, and we generated responses and auxiliary files with RMFGEN-1.53.5 and ARFGEN-1.66.4 respectively. Subsequent spectral analysis was carried out in XSPEC.

Recently, Mahdavi et al (2005) argued that the source C does not belong to Abell 194 but rather is a background cluster of galaxies at $z \simeq 0.15$. Its central galaxy is at $\alpha=01^{h} 25^{m} 35^{\mathrm{s}} .9$ $\delta=-01^{\circ} 25^{\prime} 45^{\prime \prime}$, and appears much fainter than the other cluster galaxies. The identification of source $\mathrm{C}$ with a background $\mathrm{X}$ ray source could make the linearity of the cluster a questionable property. We used the processed XMM-Newton data to derive the $\mathrm{X}$-ray properties of this source, in order to investigate further the possibility of it being a background cluster. To obtain its spectrum, we accumulated photons in a circular aperture centred on the peak of its X-ray emission and extending out to 1 arcmin. We fitted the (0.5-5.0) keV spectrum with an absorbed mekal model. The absorbing column $\left(N_{\mathrm{H}}\right)$ was fixed to the Galactic column for the direction of Abell $194\left(N_{\mathrm{H}, \mathrm{G}}=3.78 \times 10^{20} \mathrm{~cm}^{-2}\right)$. The plasma temperature $(k T)$, abundances $(Z)$, and normalization were left free to vary during the fitting procedure. Additionally, the redshift was fixed to $z=0.15$, as indicated by the work of Mahdavi et al. (2005). We obtained a temperature of $k T=1.64_{-0.18}^{+0.14} \mathrm{keV}$ and a metal abundance of $Z=0.48_{-0.18}^{+0.27} \mathrm{Z}_{\odot}$ for $\chi^{2} / d o f=113 / 118$. Adding a power-law component does not change the best fitting model. The bolometric X-ray luminosity implied by the best-fitting model for a source redshift of 0.15 is $L_{x} \simeq 0.8 \times 10^{43} \mathrm{erg} \mathrm{s}^{-1}$, which would make it consistent with observed cluster temperature-luminosity relations (Xue \& Wu 2000, Osmond \& Ponman 2004). If instead we fix the redshift to that of Abell 194 we find a temperature of $k T=$ $1.06_{-0.06}^{+0.06} \mathrm{keV}$ and a metal abundance of $Z=0.13_{-0.04}^{+0.05} \mathrm{Z}_{\odot}$, and obtain a marginally poorer fit $\left(\chi^{2} / d o f=127 / 118\right)$. We cannot therefore rule out the possibility that source C is at the redshift of A194 based purely on the X-ray data, but they are also certainly consistent with it being a background cluster, and given the results of Mahdavi et al. we exclude it from the discussion that follows.

Source A (see Fig. 1) is the dumb-bell galaxy NGC 545/7 (Fig.2). The member galaxies, NGC 545 and NGC 547 are of similar magnitudes, have recession velocities $(5338 \pm 7) \mathrm{km} \mathrm{s}^{-2}$ and $(5468 \pm 6) \mathrm{km} \mathrm{s}^{-1}$ respectively (NED), and are separated spatially by a projected distance of $\sim 30 \mathrm{arcsec}=10 \mathrm{kpc}$. Although their close separation and similar velocities make it very likely that they are bound and moving within a common gravitational potential, past observations have not revealed strong evidence of interactions in the form of tidal tails and/or bridges between the two (Fasano, Falomo, \& Scarpa 1996). The host of the WAT radio galaxy is the SE member of the pair, the galaxy NGC 547.

Source B is identified with the galaxy NGC 541, which hosts the NAT radio galaxy PKS 0123-016A. This radio galaxy may have been responsible for triggering star formation in the nearby 'Minkowski's object' (Brodie et al. 1985, van Breugel et al. 1985, Croft et al. 2006). Its recessional velocity is very close to that of the dumb-bell system $\left[(5422 \pm 6) \mathrm{km} \mathrm{s}^{-1}\right.$ (NED)], and the galaxies are separated by $\sim 4.5$ arcmin $=97.5 \mathrm{kpc}$ in projection.

Given the small differences of their velocities and separation 


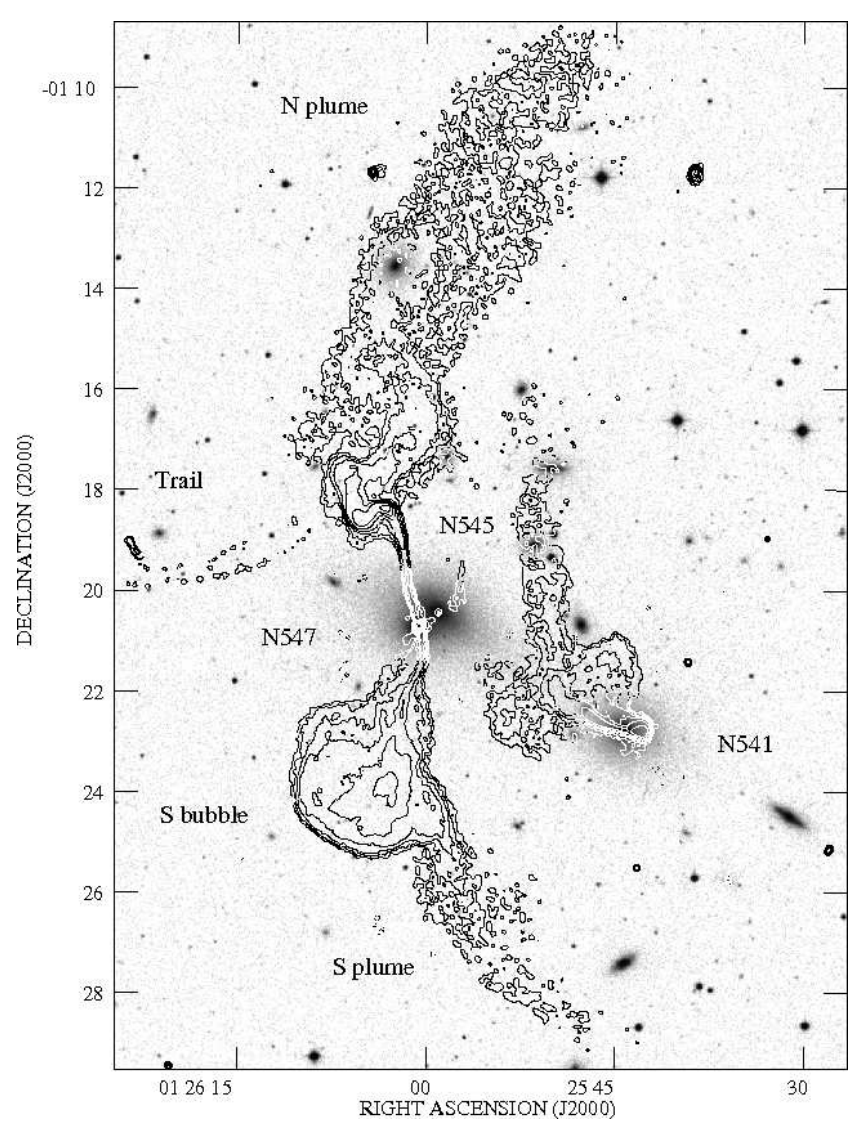

Figure 2. A $5.6 \times 5.2$ arcsec resolution 1.5-GHz VLA map superposed on the DSS2 $R$-band image of the field of 3C 40 . Grey levels are logarithmic. Contours are at $300 \times(1,2,4 \ldots) \mu \mathrm{Jy}_{\text {beam }}{ }^{-1}$. The three most massive galaxies of the cluster are labelled: note that each is a radio source, though the source associated with NGC 545 is very weak. Features of the NGC 547 WAT are also labelled.

it might appear likely that the dumb-bell galaxy and NGC 541 constitute a bound system, possibly orbiting around a common centre of gravity. Optical observations reveal an optical 'bridge' that connects NGC 545/547 and NGC 541 (see fig. 4 in Croft et al. 2006), which could be a sign of past and/or on-going interactions between them. The issue of the interactions and dynamics of these galaxies is discussed further in Section 4.

For the purpose of any subsequent spatial analysis, we firstly created exposure-corrected images for each EPIC instrument in the (0.5-5.0) keV energy range. The exposure correction was performed as in Sakelliou \& Ponman (2004). These images from each $X M M-N e w t o n$ camera were fitted in SHERPA with a 2-dimensional $\beta$-model. We also added a 2-dimensional constant to represent the background, which was not subtracted from the images. The fit was restricted to the inner 10 arcmin of the image, and all bright point sources were subtracted. We also subtracted the sources A, B, \& C (Fig. 1) by masking out circular regions with radius of 1 arcmin around them. The images from the three XMM-Newton cameras were fitted simultaneously. The core radius $\left(r_{\mathrm{c}}\right)$, the $\beta$ parameter and the location of the X-ray centre were linked and left free, so that their values are determined by the fitting procedure. The normalizations of the $\beta$-models were free to vary independently for each instrument. This procedure resulted in the following best fitting parameters: $r_{\mathrm{c}}=5.14_{-0.0924}^{+0.133} \operatorname{arcmin}=111.40_{-0.195}^{+0.199} \mathrm{kpc}$, and $\beta=1.11 \pm 0.19$. The $\mathrm{X}$-ray centre is found to lie at $\alpha_{c e n}=$ $01^{h} 25^{m} 50$ s.70 $\delta_{\text {cen }}=-01^{\circ} 22^{\prime} 10^{\prime \prime} .80$, which does not coincide with any of the bright cluster galaxies.

Source spectra were extracted in a circular region centred near the cluster centre found in the 2-dimensional analysis, and extending out to 5 arcmin. The background was taken from an annular region adjacent to the source region, between 5 and 8 arcmin from the cluster centre. Point sources, the emission around the galaxies $\mathrm{A}$ and $\mathrm{B}$, and source $\mathrm{C}$ (see Fig. 1) were excluded. Spectra from the three XMM-Newton instruments were fitted simultaneously with a mekal model modified by the absorbing column $\left(N_{\mathrm{H}}\right)$. During the fitting procedure, the $N_{\mathrm{H}}$ and metallicity $(Z)$ were held fixed to the Galactic value $\left(N_{\mathrm{H}}=N_{\mathrm{H}, \mathrm{G}}=3.78 \times 10^{20} \mathrm{~cm}^{-2}\right)$ and $Z=0.3 Z_{\odot}$ respectively. The temperature of the plasma $(k T)$, and the normalization were free to vary. We found a temperature of $k T=2.87_{-0.29}^{+0.33}\left(\chi^{2} /\right.$ d.o.f. $\left.=822.3 / 695\right)$. The temperature we derived is in good agreement with the ASCA results (Fukazawa et al. 1998), but larger than the value of $(1.36 \pm 0.04) \mathrm{keV}$ found by Mahdavi et al. (2005). In order to compare with the $L_{\mathrm{x}}-T$ relation of similar clusters we extrapolated the luminosity of the above model out to $R_{500}$. We find a bolometric luminosity of $L_{\mathrm{x}}\left(R_{500}\right) \sim 2 \times 10^{43} \mathrm{erg} \mathrm{s}^{-1}$, which makes our value for the cluster temperature consistent with the $L_{\mathrm{x}}-T$ relation presented by Osmond \& Ponman (2004).

\section{THE RADIO SOURCE $3 C 40$}

\subsection{Radio observations}

Abell 194 hosts the bright radio source 3C 40 (PKS 0123-016). In fact $3 \mathrm{C} 40$ consists of two radio galaxies, both of which contribute to the catalogued $178-\mathrm{MHz}$ flux density of 3C 40 (e.g. Maltby, Matthews \& Moffet 1963). The brighter source, which is usually known as 3C 40 but should properly be referred to as $3 \mathrm{C} 40 \mathrm{~B}$, (0123-016B) is a WAT (see Hardcastle \& Sakelliou 2004 for a definition) hosted by NGC 547, while the better-known 3C 40A (0123-016A) is a NAT hosted by NGC 541, and was studied in the radio by van Breugel et al. (1985) and Brodie et al. (1985). Detailed radio studies of the whole cluster have not been carried out to date, but images of the field have been presented by Brodie et al. (1985) and O'Dea \& Owen (1985: VLA snapshot data) and by Nikogyossian et al. (1999: NVSS data). Our attention was originally drawn to the cluster, part of the sample of Jetha, Hardcastle \& Sakelliou (2006), because the WAT 3C 40B is clearly not at the cluster centre as defined by the diffuse $\mathrm{X}$-ray emission, unlike almost all other WATs we have studied. Location at the centre of a cluster has been thought to be a key feature determining WAT morphology (e.g. Hardcastle 1998) and so it is important to understand why 3C 40 is capable of supporting a non-cluster-centre WAT.

To investigate the relationship between the complex structure of the radio galaxies and the X-ray emitting ICM we retrieved deep VLA imaging data at $330 \mathrm{MHz}$ and $1.5 \mathrm{GHz}$ from the VLA archive. Details of the VLA observations are given in Table 1 the $1.5-\mathrm{GHz}$ observations are the same as those presented by Jetha et al. (2006). Observations at higher frequencies exist, but because of the large angular scale of the source ( $24 \mathrm{arcmin})$ they cannot map the largescale structure adequately. We used archival VLA data at $4.9 \mathrm{GHz}$ (Table 1) solely in order to make accurate measurements of the bending of the jets. In addition, we observed 3C 40 with the GMRT at $240 \mathrm{MHz}$ and $610 \mathrm{MHz}$ (in dual-band mode) for 8 hours on $2006 \mathrm{Jul} 21$. The data were reduced in the standard manner within AIPS, with particular care being taken to flag data affected by radiofrequency interference (RFI) at low frequencies. At all frequencies 
Table 1. VLA archival observations of $3 \mathrm{C} 40$ used in this paper

\begin{tabular}{lllll}
\hline $\begin{array}{l}\text { Frequency } \\
(\mathrm{GHz})\end{array}$ & Obs. date & $\begin{array}{l}\text { VLA obs. } \\
\text { ID }\end{array}$ & Config. & $\begin{array}{l}\text { Time on } \\
\text { source } \\
\text { (minutes) }\end{array}$ \\
\hline 4.9 & 1984 Jan 08 & AV102 & B & 156 \\
4.9 & 1984 Jun 02 & AV102 & C & 212 \\
1.5 & 1984 Jan 08 & AV102 & B & 335 \\
1.5 & 1984 Jun 02 & AV102 & C & 206 \\
1.5 & 1985 Jul 31 & AV112 & D & 81 \\
0.33 & 1994 Aug 16 & AE97 & B & 316 \\
0.33 & 1994 Nov 20 & AE97 & C & 40 \\
\hline
\end{tabular}

below $1.5 \mathrm{GHz}$ the data were taken in spectral line mode, and (although spectral channels were averaged before imaging) multiple channels were retained throughout the analysis: imaging was carried out using the task imagr with evenly-spaced grids distributed over the primary beam to allow the removal of background point sources, and one or two iterations of phase self-calibration were used before the final maps were produced. The full-resolution 1.5$\mathrm{GHz}$ VLA map has a resolution of $5.6 \times 5.2 \mathrm{arcsec}$ (resolutions here are the FWHM of the elliptical, or, where only one number is quoted, circular restoring Gaussians) and an off-source noise of 60 $\mu \mathrm{Jy}_{\text {beam }^{-1}}$ : the $330-\mathrm{MHz}$ map has a resolution of $22 \times 19$ arcsec and an off-source noise level of $1.7 \mathrm{mJy}^{\text {beam }}{ }^{-1}$. In general, the off-source noise in the GMRT maps is good (at $240 \mathrm{MHz}$, the nominal level is $0.8 \mathrm{mJy}$ per $19 \times 15$ arcsec beam) but the image fidelity is not as good as in the VLA images, with more 'lumpiness' in the maps: we attribute this partly to residual RFI and partly to the poorer $u v$ plane coverage of the GMRT at the declination of 3C 40. The GMRT data, however, are adequate to make lowresolution maps for spectral index comparisons.

Fig. 2 shows the full-resolution 1.5-GHz VLA image superposed on the DSS-2 $R$-band optical image. This image illustrates several interesting features of the radio source. Note in particular the weak radio source associated with NGC 545, with a short jet that crosses the much more prominent jet of NGC 547, and the double-peaked trail that extends $\mathrm{E}$ from the N plume of the NGC 547 WAT (discussed in more detail in Section 4.1. In Fig. 3 we show maps at all four frequencies, made with a common resolution of 20 arcsec. These maps are made from the images used for spectral index analysis. It can be seen that all images sample the same structure (though weak negative contours at 610 and $1540 \mathrm{MHz}$ suggest that there may be some slight undersampling on the largest scales) but that the VLA data give generally smoother and more physically plausible maps.

\subsection{The WAT \& NAT hosts}

The jet and plume morphologies of the radio galaxies suggest that their host galaxies are moving relative to the ICM. Such motions relative to the ICM could result in the stripping of their interstellar media (ISMs). However, the images make clear that there is smallscale X-ray emission associated with both NGC 547 and NGC 541. In order to investigate if they retain massive ISMs, we obtained the X-ray spectra of the WAT and NAT hosts within circular regions with radii of $40 \mathrm{kpc}=110$ arcsec. For each galaxy, the background was taken in an annulus adjacent to the source region extending from 110 to 130 arcsec, and we masked out background point sources and (in the case of NGC 547) the X-ray emission associated with the companion galaxy. The spectra were modeled in XSPEC by mekal+power law models absorbed by the Galactic column, where the power-law component takes account of the expected unabsorbed jet-related nuclear emission from the FRI radio galaxies. The best-fitting temperatures were well determined and significantly lower than the temperature for the cluster as a whole $(0.61 \pm 0.03) \mathrm{keV}$ for NGC 547 and $(0.95 \pm 0.07) \mathrm{keV}$ for NGC 541. From the normalization of the mekal models (with fixed abundance of 0.3 solar) and assuming that the ISM is spherically symmetric, we find that $M_{\mathrm{ISM}, \mathrm{WAT}}=4.50_{-0.20}^{+0.12} \times 10^{9} M_{\odot}$, and $M_{\text {ISM,NAT }}=3.98_{-0.39}^{+0.36} \times 10^{9} M_{\odot}$, values that are comparable to the gas masses of other massive elliptical galaxies (e.g., Canizares, Fabbiano \& Trinchieri 1987). In addition, we carried out fits of models consisting of a central point source and isothermal $\beta$ model (both convolved with the XMM-Newton PSF) to the radial profiles of the two galaxies within the 110 -arcsec regions, with the same masking of unrelated sources. These give plausible small core radii and $\beta$ values (both galaxies have best-fitting $\beta \approx 0.5$ ) and the masses derived by integrating the $\beta$ models are very consistent with those derived from the mekal normalization.

The masses of the ISMs we found above, indicate that both the WAT and NAT hosts still retain large amounts of their ISM. It seems, that ram pressure has not been efficient in stripping them from their hot haloes.

\section{DISCUSSION}

\subsection{Spectral ageing and the 'trail'}

Both of the powerful radio sources in Abell 194 show clear radio spectral steepening along their length, as is generally observed in FRI radio sources. The spectral steepening is normally attributed to the progressive loss of high-energy electrons via synchrotron and inverse-Compton losses. However, using the radio spectra to determine the time since the electrons at various points in the plume had a flat spectrum, the 'spectral age', is well known to be fraught with difficulties: the method assumes that there is no diffuse particle acceleration throughout the plume, it requires the assumption of a model for electron diffusion (e.g. pitch angle diffusion, Jaffe \& Perola 1973), it requires that we assume a single electron energy spectrum (whereas there is some evidence for multi-component structures in the plumes of some FRI sources: Katz-Stone \& Rudnick 1997, Hardcastle 1999), and it requires that we know the magnetic field strength and, in general, the history of the magnetic field strength as seen by the electrons. In regions where the field is sufficiently weak that the magnetic field energy density is much less than that of the CMB, $B^{2} / 2 \mu_{0} \ll 8 \pi^{5}\left(k T_{\mathrm{CMB}}\right)^{4} / 15(h c)^{3}$, though, losses to inverse-Compton scattering can be presumed to dominate and the last of these requirements is removed.

In spite of these problems it is worthwhile to estimate the spectral ages of parts of the radio source in $3 \mathrm{C} 40$. While the numerical values of age that we derive will be model-dependent, they nevertheless provide a useful comparison to other ages of the system derived from dynamical constraints. In addition, we can use the ages to help us understand the structure of the radio source.

We measured flux densities from relatively large regions of the radio sources, and fitted Jaffe \& Perola (1973) aged synchrotron spectra to them, assuming an electron injection index $p$ [where $\left.N(E) \propto E^{-p}\right]$ of 2.1 (as recently argued by Young et al. 2005). We assumed that the magnetic field had the equipartition value, modelling the emission region as a cylinder in the plane of the sky. In the outer parts of the plumes of the WAT, where the spectrum 

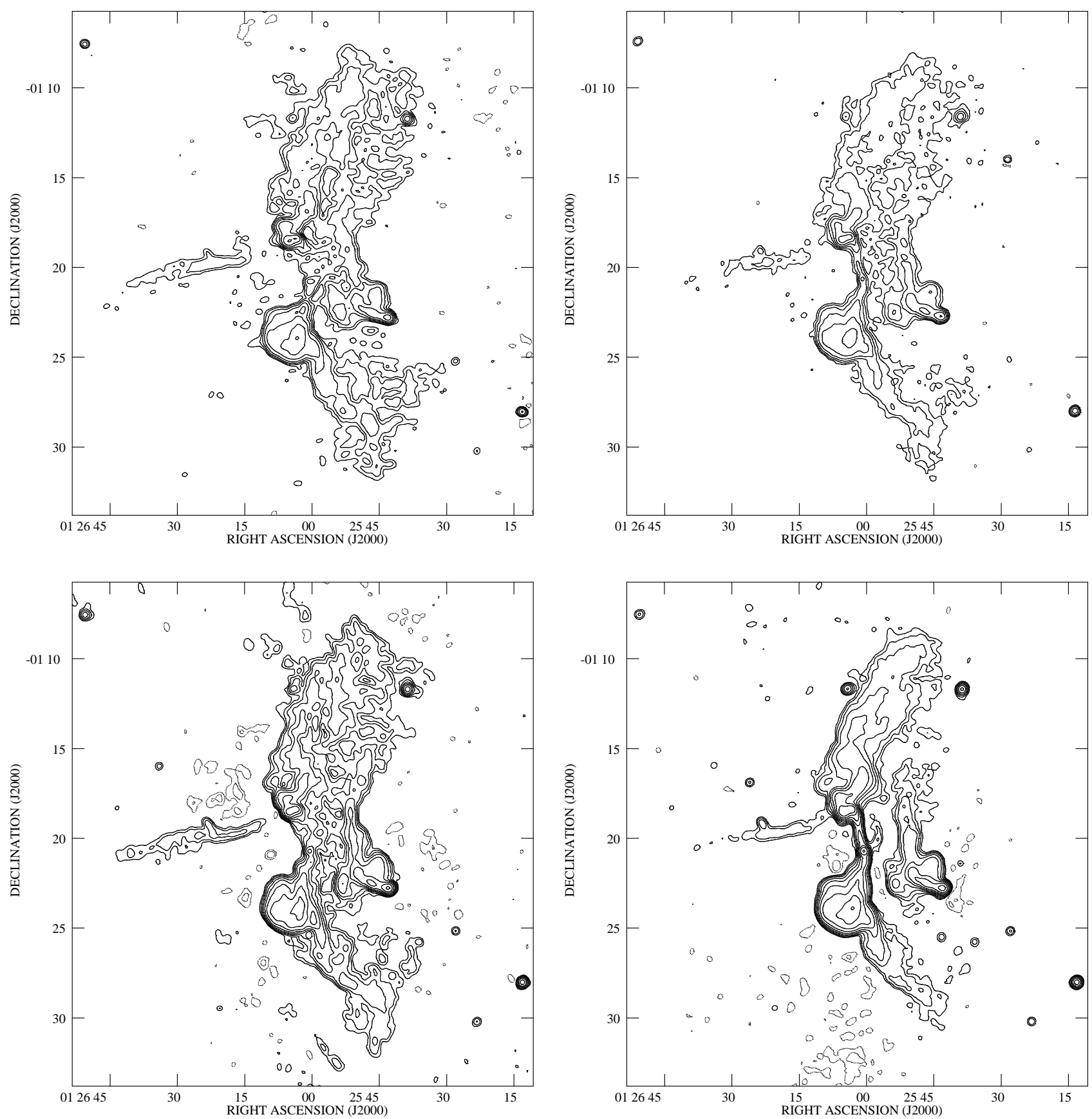

Figure 3. 3C 40 at 20-arcsec resolution. Top left: 240-MHz GMRT data. Top right: 330-MHz VLA data. Bottom left: 610-MHz GMRT data. Bottom right: 1.5-GHz VLA data. Contours are at $(-2,-1,1,2,4 \ldots)$ times the $5 \sigma$ off-source noise level on each map: these $5 \sigma$ levels are respectively $5.0,5.8,1.1$ and 0.59 mJy beam $^{-1}$.

is steepest, the equipartition magnetic field energy density is well below the CMB photon energy density, so that inverse-Compton losses dominate. Thus, the age that we derive by this method for the outer part of the WAT plumes, $1.5 \times 10^{8}$ years, is only weakly dependent on the magnetic field used (going roughly as $B^{1 / 2}$ ) and so is very little influenced by moderate departures from equipartition. This value of the age is essentially an upper limit, since the ageing $B$-field may have been higher (if equipartition is maintained, the $B$-field certainly was higher in the brighter, higher-pressure regions of the plume) and since we take no account of the effects of adiabatic expansion of the plumes, which mimics ageing. We can thus say that the time since the last particle acceleration event is $<1.5 \times 10^{8}$ years: if particle acceleration occurred predominantly at the plume base, then the material at the ends of the plume has taken $<1.5 \times 10^{8}$ years to travel there, implying travel speeds of $>1400 \mathrm{~km} \mathrm{~s}^{-1}$ (where the lower limit on speed takes into account both the limit on age and the effects of projection).

Spectral ageing analysis also sheds some light on the origin of the 'trail' seen to the E of the N plume of the WAT. The spectral index of this material is much steeper than that of the region 
Sakelliou, Hardcastle, \& Jetha

of the WAT immediately adjacent to it: this implies that it is not directly related to the WAT plume. The spectral index of the trail steepens with increasing distance eastward, and the spectral age of the steepest-spectrum material (which should be interpreted as for the WAT) is $1.4 \times 10^{8}$ years. The difference between the spectra of the trail and the adjacent WAT material, together with the doublepeaked radio structure of the trail, strongly suggests that the trail is not related to the WAT. Instead it must originate in one of the other two radio galaxies in the system. The spectral index of the NAT material on the opposite side of the WAT to the trail is comparable to - in fact slightly steeper than - that of the material at the $\mathrm{E}$ end of the trail, and there is some evidence in the radio maps that the plume extending to the N of the NAT has the same doublepeaked structure as the trail. In this picture the material produced by the NAT has to make a sharp $\left(90^{\circ}\right)$ bend in projection to form the trail. The alternative is that the trail was produced by the weak radio source that we associate with NGC 545, with the connection between the host galaxy and the trail being obscured by the bright intervening WAT emission. The lack of any obvious connection in the radio between NGC 545 and the trail argues against this idea, however.

\subsection{Dynamics of the cluster centre}

All the data presented in the previous sections show that the core region of Abell 194 is occupied by large galaxies. These galaxies are currently in close separation, they host bent radio sources, and live in an elongated X-ray envelope. Taking into account these observational facts, and past beliefs that the existence of tailed radio galaxies in clusters is closely connected to the disturbances induced by recent cluster mergers, as more tailed sources are found in clusters that have suffered a recent merger event (e.g., Bliton et al. 1998), one could naturally think that Abell 194 has been recently assembled by the accretion of smaller mass units. In such a formation scenario the radio galaxies could be newcomers to the cluster, having fallen into it from its outskirts; now they should be crossing its central region at high speeds. If this picture is correct, their radio jets should be bent by the resulting high ram pressure. It is also accepted that they should not retain large amounts of ISMs, as they should have been stripped during their in-fall towards the cluster centre. Luckily, we can test all the above using the information from the X-ray and radio data.

The shape of the jets, for example, puts constraints on the galaxy velocities, if we assume that the jets of both the NAT and the WAT are bent by ram pressure, and apply the usual equation: $\rho_{\mathrm{j}} v_{\mathrm{j}}^{2} / R=\rho_{\mathrm{ICM}} v_{\text {gal }}^{2} / h$, where $h$ is the scale height of the jet and $R$ is its radius of curvature. The structure of the NAT clearly suggests bending by bulk motion. The WAT jets, though bent, are less obviously symmetrically swept backwards, but we estimate the possible effects of bending from the prominent bend in the $\mathrm{S}$ jet between 15 arcsec and 1 arcmin from the nucleus. We measured the scale heights $\left(h_{\mathrm{NAT}}, h_{\mathrm{WAT}}\right)$ and radii of curvature $\left(R_{\mathrm{NAT}}, R_{\mathrm{WAT}}\right)$ from high-resolution radio maps, obtaining $h_{\mathrm{NAT}}=4 \operatorname{arcsec}, h_{\mathrm{WAT}}=$ $1 \operatorname{arcsec}, R_{\mathrm{NAT}}=37 \operatorname{arcsec}, R_{\mathrm{WAT}}=65 \operatorname{arcsec}$. For the density of the ICM near the location of both radio galaxies we used the $\beta$ model fits described above and the methods of Birkinshaw \& Worrall (1993) to determine a value of $n_{\mathrm{p}, \mathrm{ICM}}=5 \times 10^{-4} \mathrm{~cm}^{-3}$ : the dominant uncertainty in this quantity is the systematic uncertainty from the assumed abundance, but for a plausible abundance range at our best-fitting cluster temperature the fractional uncertainty is of the order $20 \%$. The most uncertain quantities in the above equation are the densities and velocities of the jets. Lower limits on the den- sities may be estimated from the minimum energy density, which is $7 \times 10^{-13} \mathrm{~J} \mathrm{~m}^{-3}$ for the NAT and $3 \times 10^{-12} \mathrm{~J} \mathrm{~m}^{-3}$ for the WAT. Since there is no sign of beaming in the NAT jet, we take $v_{\mathrm{j}, \mathrm{NAT}}=0.1 c$, a reasonable value for the jet speed in FRI jets after deceleration (Laing \& Bridle 2002) while for the WAT we take $v_{\mathrm{j}, \mathrm{WAT}}=0.5 c$, the best-fitting value for WAT jet speeds derived by Jetha et al. (2006), and assume the jet is in the plane of the sky. This gives limits of $v_{\text {gal,NAT }}>30 \mathrm{~km} \mathrm{~s}^{-1}$ and $v_{\text {gal,WAT }}>110$ $\mathrm{km} \mathrm{s}^{-1}$. These are lower limits because we do not know the true jet densities. For NATs, like other normal twin-jet FRIs, we expect deceleration to have taken place by entrainment, so that the true density could be substantially above the minimum value. The models of Laing \& Bridle (2002), applied to the jet in 3C 31, require a jet density contrast $\left(\rho_{\text {jet }} / \rho_{\text {ICM }}\right)$ of $10^{-4}$ on $10-\mathrm{kpc}$ scales, which in NGC 541 would correspond to $v_{\text {gal, NAT }}=140 \mathrm{~km} \mathrm{~s}^{-1}$. If the speed of NGC 541 with respect to the ICM were much higher than this, the jet would have to be substantially heavier with respect to the ICM than that in $3 \mathrm{C} 31$, and/or substantially faster than seems likely, in order to avoid being more strongly bent by ram pressure.

The above calculations suggest that the speeds of the WAT and NAT hosts should be low compared to the trans- or super-sonic velocities they would have had if they have fallen recently into the cluster (see for example fig. 1 in Acreman et al. 2003). As was mentioned in Section 2 past measurements of their recessional velocities also found a low velocity separation along the line of sight.

Such low velocities are not generally adequate to strip the galaxies from their ISMs. Acreman et al. (2003) simulated the stripping process during the infall of elliptical galaxies towards the centre of a cluster. As their simulated cluster had a temperature of $\mathrm{kT}=2.7 \mathrm{keV}$, it is very comparable to Abell 194. As can be seen in their fig. 1 the galaxy falls from the outskirts of the cluster towards the cluster centre. Its velocity increases and reaches supersonic velocities $\left(\geqslant 1000 \mathrm{~km} \mathrm{~s}^{-2}\right)$ only around the cluster core region. In all cases they studied, the galaxies do not retain more than $\sim 4 \times 10^{9} M_{\odot}$ of their ISM; the gas mass that remains within these galaxies can be as low as $\sim 10^{8} M_{\odot}$. Only the ISMs of very massive galaxies are not severely stripped: galaxies with dark matter haloes as large as $M_{\text {halo }}=4 \times 10^{12} M_{\odot}$ do not suffer any substantial reduction of their ISMs. Severe stripping occurs only when the galaxy is crossing the dense cluster core cluster region at high velocities. The gas masses we calculated in Section 3.2 strongly suggest that that the radio galaxies in Abell 194 have not been stripped of their X-ray halos, and therefore they cannot be currently falling towards the cluster centre from its outskirts. Thus, the lack of stripping also argues against a scenario in which the core region of Abell 194 is undergoing a major merger event.

We are therefore forced to the conclusion that the NAT and WAT hosts are moving at low speeds. The two radio galaxies are at projected distance of $\sim 50 \mathrm{kpc}$ from the cluster centre. The gravitating cluster mass within such a radius is $\sim 1 \times 10^{12} \mathrm{M}_{\odot}$ (Nikogossyan et al. 1999). If they are bound their velocities should be $\leqslant 300 \mathrm{~km} \mathrm{~s}^{-1}$, which is within the allowed limits of the galaxy velocity found above. Thus, we conclude that they are bound to, and probably rotating around, the cluster centre. The expected orbital speed is adequate to bend the jets while still retaining the observed amount of hot gas within the galaxies.

It should also be noted that there is nothing unusual about the distribution of the cluster gas. The X-ray data do not show any signs of features expected from a recent cluster merger event, such as regions of enhanced emission due to shock waves or small in-falling groups of galaxies.

Thus it appears that these cluster galaxies are most likely or- 
biting around the common centre of mass inside the cluster core. The tips of the radio galaxies are probably not passive radio trails left behind in the cluster as the galaxies have moved to their current positions. It would have taken $1.2 \mathrm{Gyr}$ for the NAT, for example, to create the North trail as a passive trail (assuming motion of 6 arcmin, or $130 \mathrm{kpc}$, at $100 \mathrm{~km} \mathrm{~s}^{-1}$ ) and this is inconsistent with the observed spectral ages (which, as discussed above, should probably be taken as upper limits). Similarly, the initially attractive idea that the bending of the plumes of the WAT (in the opposite sense to the current bending of the inner jets) is a result of their having being produced at a stage when the host galaxy was moving in the opposite direction is inconsistent with the very long orbital period of the system (the current period is $T \simeq 1 \mathrm{Gyr}$ ), unless there is substantial particle reacceleration in the plumes. In our picture, in which the host galaxies are moving slowly and have remained close to the cluster centre for much longer than the radio source lifetimes, the bending of both WAT and NAT plumes must be a result of the interaction between the plume flow (transport speeds must still be substantial if the observed spectral ages are accurate, see above) and its environment. As discussed by Hardcastle, Sakelliou \& Worrall (2005) interactions between a fast plume and external material with sub-sonic speeds can only have a significant effect if the plume is very light compared to the external medium, and in this case buoyant forces probably also play an important role.

\section{SUMMARY AND CONCLUSIONS}

We have presented XMM-Newton, VLA, and GMRT observations of the nearby cluster Abell 194. Its core region is occupied by the WAT 0123-016B, and the NAT 0123-016A, which together comprise the radio source $3 \mathrm{C} 40$.

The X-ray data do not show any signs of features expected from recent cluster merger activity, like regions of enhanced emission due to shock waves or small in-falling groups of galaxies. The $\mathrm{X}$-ray and radio data argue that the jets and plumes of the radio sources do not require high galaxy velocities to be bent into their current shapes. Relatively low velocities of the order of a few hundred $\mathrm{km} \mathrm{s}^{-1}$ are adequate to produce the bending. Additionally, both galaxies retain a large fraction of their ISM, suggesting that they are not severely stripped as it would have been the case if they are newcomers to the cluster having fallen from its outskirts. The WAT's host is not at the cluster centre as defined by the distribution of the ICM.

It appears that Abell 194 is not suffering a major merger event, that would involve large relative galaxy velocities. A likely scenario is that the radio galaxies are rotating around the cluster centre in the core region of Abell 194. Such a motion could explain the observational facts like the bending of the jets/plumes of the radio galaxies, the presence of the optical bridge, and the lack of stripping of the galaxy haloes. The presence of the two massive X-ray haloes and the lack of rapid motions of the host galaxies probably explain how there can be two powerful radio galaxies so close to the centre of the cluster and why $3 \mathrm{C} 40 \mathrm{~B}$ can have a classical WAT morphology in spite of its offset from the cluster centre.

\section{ACKNOWLEDGMENTS}

We thank an anonymous referee for constructive comments that helped us to improve the paper. The Digitized Sky Survey (DSS), and the NASA/IPAC Extragalactic Database (NED) have been used. The present work is based on observations obtained with XMM-Newton, an ESA science mission with instruments and contributions directly funded by ESA Member States and the USA (NASA). The National Radio Astronomy Observatory is a facility of the National Science Foundation operated under cooperative agreement by Associated Universities, Inc. We thank the staff of the GMRT for their help with the observations with that telescope: GMRT is run by the National Centre for Radio Astrophysics of the Tata Institute of Fundamental Research, India. IS acknowledges the support of the European Community under a Marie Curie IntraEuropean Fellowship. MJH thanks the Royal Society for a research fellowship.

\section{REFERENCES}

Acreman D.M., Stevens I.R., Ponman T.J., Sakelliou I., 2003, MNRAS, 341, 1333

Birkinshaw, M., Worrall, D.M., 1993, ApJ, 412, 568

Bliton M., Rizza E., Burns J.O., Owen F.N., Ledlow M.J., 1998, MNRAS, 301, 609

Brodie J.P., Bowyer S., McCarthy P., 1985, ApJ, 293, 59

Burns J.O., Rhee G., Owen F.N., Pinkney J., 1994, ApJ, 423, 94

Canizares C.R., Fabbiano G., Trinchieri G., 1987, ApJ, 312, 503

Croft S., van Breugel, W., de Vries W., Dopita M., Martin C., Morganti R., Neff S., Oosterloo T., Schiminovich D., Stanford S. A., van Gorkom J., 2006, ApJ, 647, 1040

Ebeling H., Voges W., Böhringer H., Edge A.C., Huchra J.P., Briel U.G., 1996, MNRAS, 281, 799

Hardcastle M.J., 1998, MNRAS, 298, 569

Hardcastle M.J., 1999, A\&A, 349, 381

Hardcastle M.J., Sakelliou I., 2004, MNRAS, 349, 560

Hardcastle M.J., Sakelliou I., Worrall D.M., 2005, MNRAS, 359, 1007

Jaffe W.J., Perola G.C., 1973, A\&A, 26, 423

Jetha N.N., Hardcastle M.J., Sakelliou I., 2006, MNRAS, 368, 609

Jetha N.N., Sakelliou I., Hardcastle M.J., Ponmman T.J., Stevens I.R., 2005, MNRAS, 358, 1394

Katz-Stone D.M., Rudnick L., 1997, ApJ, 488, 146

Laing R.A., Bridle A.H., 2002, MNRAS, 336, 328

Lazzati D., Campana S., Rosati P.., Chincarini G., Giacconi R., 1998, A\&A, 331, 41

Mahdavi A., Finoguenov A., Böhringer H., Geller M.J., Henry J.P., 1005, ApJ, 622, 187

Maltby P., Matthews T.A., Moffet A.T., 1963, ApJ, 137, 153

Nikogossyan E., Durret F., Gerbal D., Magnard F., 1999, A\&A, 349, 97

O’Dea C.P., Sarazin C.L., Owen F.N., 1987, ApJ, 316, 113

O’Dea C.P., Owen F.N., 1985, AJ, 90, 927

Osmond J.P.F., Ponman T.J., 2004, MNRAS, 350, 1511

Pinkney J., Burns J.O., Hill J.M., 1994, AJ, 108, 2031

Sakelliou I., Merrifield M.R., 2000, MNRAS, 311, 649

van Breugel W., Filippenko A. V., Heckman T., Miley G., 1985, ApJ, 293, 83

Xue Y-J, Wu X-P, 2000, ApJ, 538, 65

Young A., Rudnick L., Katz D., DeLaney T., Kassim N.E., Makishima K., 2005, ApJ, 626, 748 